\title{
Métodos de Pré-codificação e de Alocação de Potência em Sistemas Cell-free
}

\author{
Thaís C. Sampaio, Maria Clara R. Lobão, e Yuri C. B. Silva.
}

Resumo-Considerando a evolução dos sistemas de comunicações móveis, o conceito de comunicação cell-free surge como uma oportunidade de se alcançar maiores taxas e utilizar os recursos de forma mais eficiente. Este estudo realiza uma análise, por meio de simulações computacionais, de técnicas de pré-codificação e de alocação de potência, demonstrando que é possível obter ganhos mediante seu uso combinado em cenários cell-free.

Palavras-Chave-MIMO, cell-free, alocação de potência, précodificação.

\section{INTRODUÇÃo}

Durante a quinta geração (fifth generation, 5G), com o crescente número de usuários e a necessidade de fornecer altas taxas de dados, o uso de múltiplas entradas múltiplas saídas (multiple-input multiple-output, MIMO) em cenários cell-free [1] surge como uma alternativa capaz de aprimorar a transmissão de dados, latência e integridade do sinal. Essa escolha oferece também melhor desempenho quando comparado a uma rede MIMO multicelular, de acordo com estudos recentes [2]. O objetivo deste estudo é a análise desse cenário considerando métodos diferentes de pré-codificação e de alocação de potência, comparando as taxas e as relações sinal-ruído por meio de implementações computacionais e utilizando como base referências recentes relacionadas ao tema. Os resultados obtidos revelam coerência com a literatura.

\section{Modelo Do Sistema}

Consideramos uma rede que possui $N_{a}$ pontos de acesso (access points, APs) e $N_{u}$ terminais móveis (user equipments, UEs), na qual cada usuário possui uma única antena e cada AP está equipado com $N_{t}$ antenas. Nesse cenário, todos os APs se comunicam com todos os usuários no sistema. Modelamos o canal de propagação $\mathbf{h}_{k, a} \in \mathbb{C}^{N_{t}}$ entre o $k$-ésimo usuário e o $a$-ésimo AP como

$$
\mathbf{h}_{k, a}=\sqrt{\beta_{k, a}} \mathbf{g}_{k, a}
$$

na qual $\beta_{k, a}$ e $\mathbf{g}_{k, a}$ correspondem, respectivamente, ao coeficiente da perda de percurso e sombreamento e aos coeficientes de pequena escala entre o $a$-ésimo $\mathrm{AP}$ e o $k$-ésimo usuário. Assume-se desvanecimento Rayleigh e o coeficiente de larga escala $\beta_{k, a}$ obedece ao modelo em [2]

$$
\beta_{k, a}=10^{\frac{\mathrm{PL}_{k, a}}{10}} 10^{\frac{\sigma_{s h} z_{k, a}}{10}},
$$

Thaís C. Sampaio, Maria Clara R. Lobão, Yuri C. B. Silva, Grupo de Pesquisa em Telecomunicações sem Fio (GTEL), Universidade Federal do Ceará (UFC), Fortaleza, Brasil. E-mails: \{thaisc, mclararodrigues\} @alu.ufc.br, yuri@gtel.ufc.br. Este trabalho foi parcialmente financiado pelo CNPq. em que $\mathrm{PL}_{k, a}$ é a perda de percurso em $\mathrm{dB}$ e $\sigma_{s h}$ e $z_{k, a}$ representam o desvio padrão e a correlação espacial do sombreamento, respectivamente. $\mathrm{O}$ vetor de canal conjunto de um usuário com relação a todos os APs é dado por $\mathbf{h}_{k}=$ $\left[\mathbf{h}_{k, 1}^{T} \ldots \mathbf{h}_{k, N_{a}}^{T}\right]^{T} \in \mathbb{C}^{N_{t} N_{a}}$, e a matriz total de canal é dada por $\mathbf{H}=\left[\begin{array}{lll}\mathbf{h}_{1} \cdots & \mathbf{h}_{N_{u}}\end{array}\right]^{T} \in \mathbb{C}^{N_{u} \times N_{t} N_{a}}$. A matriz de précodificação $\mathbf{W} \in \mathbb{C}^{N_{t} N_{a} \times N_{u}}$ é descrita na seção II-A.

\section{A. Pré-codificação}

1) Zero-forcing $(Z F)$ : Consiste na transmissão conjunta considerando a matriz total $\mathbf{H}$, na qual todos os sinais que não sejam o sinal desejado são considerados interferência e cancelados a partir da pseudo inversa da matriz de canal [3].

$$
\mathbf{W}_{\mathrm{ZF}}=\left(\mathbf{H}^{H} \mathbf{H}\right)^{-1} \mathbf{H}^{H} \text {. }
$$

2) Singular Value Decomposition (SVD): Nessa abordagem, realiza-se a decomposição em valores singulares e depois projeta-se o canal no espaço nulo seguido de projeção no espaço de sinal, para que os usuários estejam sob zero interferência e concentre-se energia no espaço útil de sinal, de forma semelhante à abordagem em [4].

3) Maximum Ratio (MR): De acordo com [1], esse é um método de baixa complexidade, apresentando desempenho quase ótimo quando há grande número de antenas no AP, com

$$
\mathbf{w}_{k, a}=\sqrt{\rho_{k, a}} \frac{\mathbf{h}_{k, a}^{*}}{\sqrt{E\left\{\left\|\mathbf{h}_{k, a}\right\|^{2}\right\}}},
$$

em que $\rho_{k, a} \geq 0$ é a potência que o AP $a$ está atribuindo para transmitir para o UE $k$.

4) Minimum Mean Square Error (MMSE): Essa abordagem minimiza o erro quadrático médio (MSE). Ela não necessariamente elimina toda a interferência inter-simbólica, mas melhora o condicionamento da matriz a ser invertida por meio do fator $\lambda$.

$$
\mathbf{W}_{\mathrm{MMSE}}=\left(\mathbf{H}^{H} \mathbf{H}+\lambda \mathbf{1}_{N}\right)^{-1} \mathbf{H}^{H} .
$$

\section{B. Alocação de Potência}

1) Alocação de Potência Proporcional (PPA): Essa técnica divide a potência máxima para o $\mathrm{AP} a, P_{B_{-} a}$, de maneira proporcional à intensidade do canal [2]. Assim, usuários com bons coeficientes de canal receberão parcela maior da potência.

$$
P_{k, a}=P_{B_{-} a} \frac{\gamma_{k, a}}{\sum_{j} \gamma_{j, a}} .
$$

Nessa expressão, $P_{k, a}$ é a potência transmitida do AP $a$ para o usuário $k$ e $\gamma_{k, a}=\mathrm{E}\left[\mathbf{h}_{k, a}^{H} \mathbf{h}_{k, a}\right]$. 
2) Alocação de Potência via Waterfilling (WF): Esse tipo de alocação é calculada atendendo à expressão em [2]:

$$
P_{k, a}=\left(\nu_{a}-L_{k, a}\right)^{+},
$$

na qual $L_{k, a}=\sigma^{2} / \gamma_{k, a}$ e $\nu_{a}$ representa o nível de potência obtido pelo algoritmo de waterfilling.

\section{Restrição de Potência}

A seleção dos vetores de pré-codificação deve satisfazer a uma restrição de potência por base [3]. Dada a matriz preliminar de transmissão $\overline{\mathbf{W}}$, já incluindo a alocação de potência descrita na seção anterior, projeta-se uma matriz de transmissão $\mathbf{T}=\overline{\mathbf{W}} \boldsymbol{\Omega}$ satisfazendo

$$
\operatorname{Tr}\left(\sum_{k=1}^{K} \mathbf{T}_{k}^{[a]} \mathbf{T}_{k}^{[a]^{H}}\right) \leq P_{B_{-} a},
$$

na qual $\mathbf{T}_{k}^{[a]}$ corresponde à matriz de transmissão do AP $a$ para o usuário $k$. A solução para o problema é dada por

$$
\Omega=\mu \mathbf{I}, \quad \mu=\min _{a=1,2, \ldots, N_{a}}\left(\frac{P_{B_{-} a}}{\left\|\overline{\mathbf{W}}^{[a]}\right\|_{F}^{2}}\right),
$$

em que $\Omega$ é uma matriz diagonal $N_{u} \times N_{u}$.

\section{ANÁLISE COMPUTACIONAL}

Para a simulação ${ }^{1}$, considera-se $N_{a}=100$ APs com quatro antenas cada e $N_{u}=60$ UEs equipados com uma única antena cada. Os APs estão espaçados uniformemente em uma malha quadrada de $1 \mathrm{~km}^{2}$ e os UEs são posicionados aleatoriamente nesta área. Cada AP transmite, no máximo, $P_{B_{a}}=200 \mathrm{~mW}$. Considera-se também $\tau=200$ sorteios de canal. Os demais parâmetros para modelagem da perda de percurso e cálculo da taxa são definidos na Tabela I. As curvas em Fig. 1 e Fig. 2 representam os resultados obtidos através das simulações.

TABELA I

PARÂMETROS DE SIMULAÇÃO.

\begin{tabular}{lc}
\hline \hline Ruído Térmico & $-174 \mathrm{dBm} / \mathrm{Hz}$ \\
Frequência & $f_{0}=1,9 \mathrm{GHz}$ \\
Largura de banda & $W=20 \mathrm{MHz}$ \\
\hline
\end{tabular}

Como pode-se ver na Fig. 1, as simulações utilizando précodificação via MMSE mostraram taxas similares às obtidas via $\mathrm{ZF}$, com o MMSE oferecendo desempenho ligeiramente melhor para baixos valores de relação sinal-ruído (signal-tonoise ratio, SNR) e convergindo para o ZF em altas SNRs, como esperado. A alocação de potência não teve impacto significativo no desempenho do ZF e MMSE, uma vez que o canal equivalente obtido por esses pré-codificadores não apresenta variação perceptível entre os diferente canais, não havendo margem para a atuação do método de alocação de potência. O MR, por sua vez, obteve o pior desempenho, pois não realizou cancelamento da interferência. Por fim, o método com pré-codificação SVD e alocação de potência via

\footnotetext{
${ }^{1}$ Implementada em Python usando a biblioteca NumPy.
}

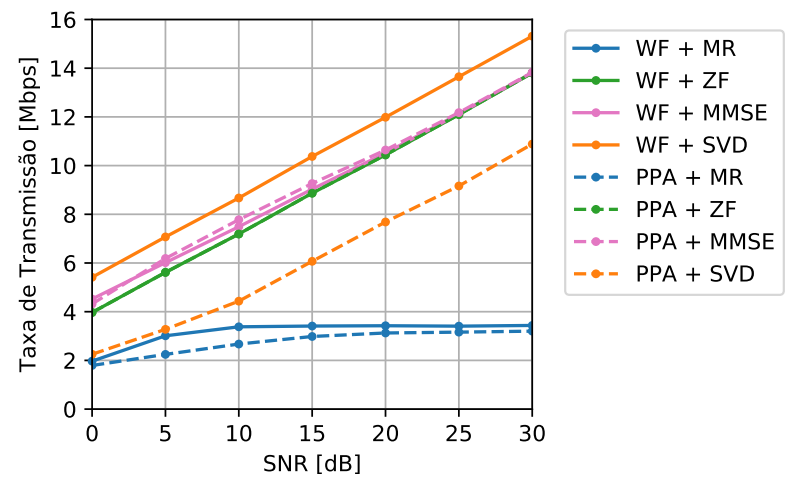

Fig. 1. Taxa de transmissão média em função da SNR para um sistema cell-free com os parâmetros de simulação da Tabela I.

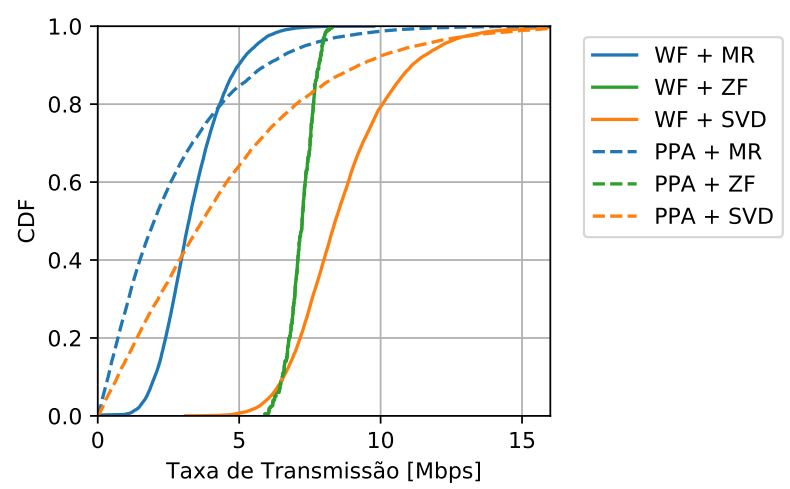

Fig. 2. Curvas de CDF da taxa de transmissão para um sistema cell-free com os parâmetros de simulação da Tabela I e SNR $=10$ dB.

WF alcançou o melhor desempenho. A Fig. 2 apresenta as curvas de CDF da taxa de transmissão, considerando uma SNR de $10 \mathrm{~dB}$. Para melhorar a visualização, não foi apresentado o resultado do MMSE, que possui um desempenho muito próximo ao ZF. Considerando os métodos de pré-codificação efetivamente impactados pela alocação de potência, no caso o MR e o SVD, percebe-se que o WF possui um desempenho bem melhor que o PPA em termos de taxa de transmissão.

\section{CONCLUSÕES}

Por meio deste estudo foi possível verificar o impacto que os mecanismos de pré-codificação e de alocação de potência possuem sobre o desempenho de um sistema cellfree. As perspectivas futuras deste estudo consideram: um maior número de antenas, tendendo para o caso massivo; consideração de aspectos práticos; aplicação de técnicas mais robustas, incluindo o aprendizado de máquina.

\section{REFERÊNCIAS}

[1] E. Björnson and L. Sanguinetti, "Scalable cell-free massive MIMO systems," IEEE Trans. Commun., vol. 68, no. 7, pp. 4247-4261, Jul. 2020.

[2] C. D'Andrea, A. Garcia-Rodriguez, G. Geraci, L. G. Giordano, and S. Buzzi, "Cell-free massive MIMO for UAV communications," in Proc. IEEE Int. Conf. on Commun. Workshops (ICC), May 2019, pp. 1-6.

[3] H. Zhang and H. Dai, "Cochannel interference mitigation and cooperative processing in downlink multicell multiuser MIMO networks," EURASIP J. on Wireless Commun. and Netw., vol. 2004, no. 2, pp. 222-235, 2004.

[4] Q. H. Spencer, A. L. Swindlehurst, and M. Haardt, "Zero-forcing methods for downlink spatial multiplexing in multiuser MIMO channels," IEEE Trans. Signal Process., vol. 52, no. 2, pp. 461-471, Feb. 2004. 